\title{
Hydrogen Network Fluctuations of Alcohols. Evidence from Ultrasonic, Dielectric, and Shear Impedance Spectrometry
}

\author{
U. Kaatze
}

Received: 4 March 2011 / Accepted: 3 June 2011 / Published online: 18 June 2011

(C) The Author(s) 2011. This article is published with open access at Springerlink.com

\begin{abstract}
Broadband dielectric spectra (1 MHz to $40 \mathrm{GHz}$ ) and ultrasonic attenuation spectra $(300 \mathrm{kHz}$ to $3 \mathrm{GHz})$ as well as frequency-dependent complex shear viscosities (6 MHz to $120 \mathrm{MHz}$ ) are reported for alcohols and for their mixtures with other alcohols, with alkanes, and with water. The spectra are discussed with a view to features controlling the molecular dynamics of associating liquids. Special attention is given to (i) the rotation of terminal single-hydrogen-bonded hydroxy groups of associated structures, (ii) the dominating relaxation process of the structures, involving multiply-hydrogen-bonded hydroxy groups, (iii) the alkyl chain rotational isomerization, (iv) effects of clustering in alcohol-alkane mixtures, and (v) indications of non-critical concentration fluctuations of alcohol-water mixtures. The hydrogen network fluctuations of the alcohol systems can be consistently described in terms of a wait-and-switch model of relaxation. The concentration fluctuations are well represented by a recent "unifying" theoretical model, indicating a predominant impact of hydrophobic groups in the promotion of a micro-heterogeneous structure of mixtures with water.
\end{abstract}

Keywords Alcohols - Concentration fluctuations - Dielectric spectroscopy · Hydrogen networks · Shear viscosity · Ultrasonic spectroscopy

\section{Introduction}

The remarkable features of liquids are predicted on the almost balanced counteraction of ordering effects from molecular interactions on the one hand and

U. Kaatze $(\bowtie)$

Drittes Physikalisches Institut, Georg-August-Universität Göttingen, Friedrich-Hund-Platz 1,

37077 Göttingen, Germany

e-mail: uka@physik3.gwdg.de 
disordering thermal agitation on the other. In this context associating liquids, with their hydrogen-bond energy on the order of thermal energy, are of particular relevance because of their extreme importance in both the biosphere and technical applications. Without any doubts, the unique properties of omnipresent water on earth are a focus of special interest. Useful insights into the hydrogen-bond fluctuations of this outstanding liquid can be derived, however, also from studies of likewise associated alcohols.

Because of their basically amphiphilic character, alcohols are favorable liquids to investigate the dynamics of hydrogen-bond networks. Alcohols possess at least one dipolar hydroxyl group offering a hydrogen-bond donating and accepting site. Additionally they hold a non-polar alkyl part, with its length and degree of branching variable across wide ranges. In investigations into the effects of hydrogen bonding, alcohols thus offer a class of liquids with easily tuneable polarity, molecular size, and shape.

Though considerable efforts have been made in the past to elucidate the properties of alcohols [1-8], many features of their structure and dynamics are still unclear. In this paper some aspects of monohydric alcohols and their mixtures with alkanes and water as well as mixtures among alcohols are summarized and discussed briefly. The focus will be results from different relaxation methods at room temperature.

\section{Experimental Results}

\subsection{Dielectric Spectra}

As an example the complex dielectric spectrum,

$$
\varepsilon(\nu)=\varepsilon^{\prime}(v)-\mathrm{i} \varepsilon^{\prime \prime}(\nu)=P(\nu) /\left(\varepsilon_{0} E(\nu)\right)+1
$$

of isopropanol is shown in Fig. 1. Here $P$ is the modulus of the electric polarization, $E$ is the electric field strength, $\varepsilon_{0}$ is the electric field constant, $v$ is the frequency, and $\mathrm{i}^{2}=-1$. At microwave frequencies the data display a dispersion $\left(\mathrm{d} \varepsilon^{\prime} / \mathrm{d} v<0\right) /$ dielectric loss $\left(\varepsilon^{\prime \prime}>0\right)$ region, indicating relaxation characteristics. Within the frequency range of measurement, the spectrum can be well represented by a sum of two Debye-type relaxation terms $(\omega=2 \pi \nu)$,

$$
\varepsilon(\nu)=\varepsilon(\infty)+A_{\mathrm{d} 1} /\left(1+\mathrm{i} \omega \tau_{\mathrm{d} 1}\right)+A_{\mathrm{d} 2} /\left(1+\mathrm{i} \omega \tau_{\mathrm{d} 2}\right)
$$

with discrete relaxation times $\tau_{\mathrm{d} 1}$ and $\tau_{\mathrm{d} 2}$ and relaxation amplitudes $A_{\mathrm{d} 1}$ and $A_{\mathrm{d} 2}$, respectively. Here $A_{\mathrm{d} 1}+A_{\mathrm{d} 2}=\varepsilon(0)-\varepsilon(\infty)$, where $\varepsilon(0)$ and $\varepsilon(\infty)$ are the permittivity values following from extrapolation to low and high frequencies. Measurements of alcohols including even higher frequencies reveal altogether three well-separated relaxation terms $[10,11]$. Often the dominating low-frequency term ("d1") is subject to a relaxation time distribution [12] such as a Davidson-Cole distribution [13], so that 
Fig. 1 Real part $\left(\varepsilon^{\prime}(v), \bullet\right)$ and negative imaginary part $\left(\varepsilon^{\prime \prime}(v), \circ\right)$ of the complex permittivity of isopropanol at $25^{\circ} \mathrm{C}$ displayed versus frequency $v$ [9]. The inset shows the data in a complex plane representation with the frequency being the parameter. Full lines show the analytical representation of the spectrum by a sum of two Debye terms (Eq. 2). Dashed lines indicate the contributions from the low-frequency relaxation term

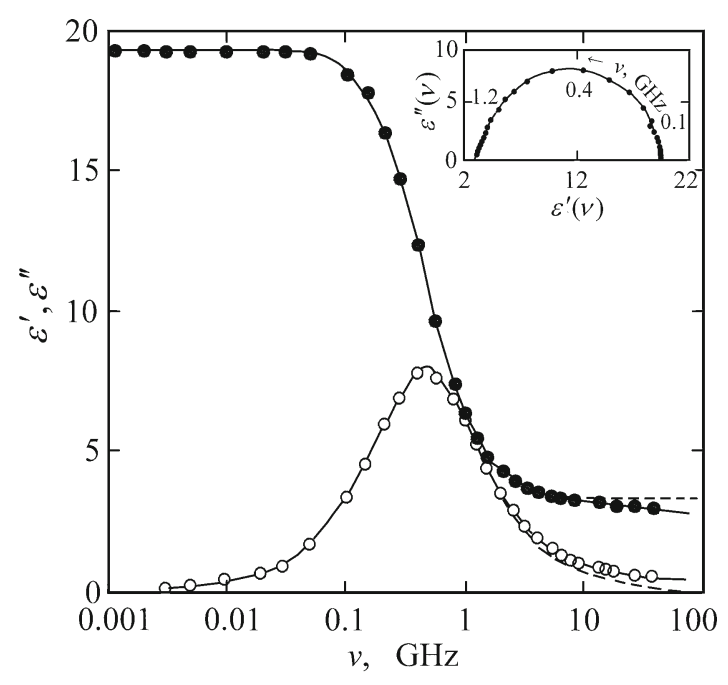

Fig. 2 Ultrasonic attenuation spectrum of $n$-dodecanol at $25^{\circ} \mathrm{C}[14]$ in the frequencynormalized format and as excess attenuation per wavelength (inset). Full lines are graphs of a two-Debye-term relaxation function. The dashed line shows the contribution from the shear viscosity when assumed to be frequency independent (Eq. 5). Dotted lines show the subdivision of the excess attenuation spectrum in two Debye terms

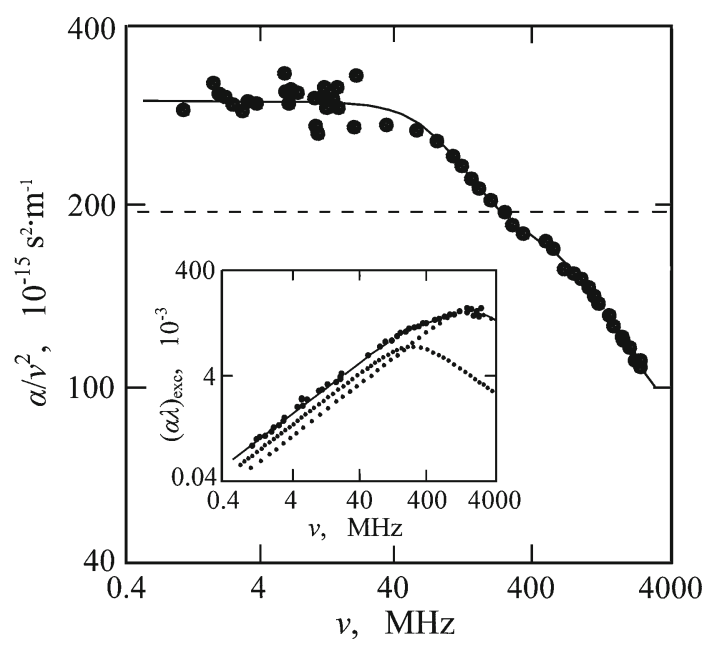

$$
\varepsilon(\nu)=\varepsilon(\infty)+A_{\mathrm{d} 1} /\left(1+\mathrm{i} \omega \tau_{\mathrm{d} 1}\right)^{(1-\beta)}+A_{\mathrm{d} 2} /\left(1+\mathrm{i} \omega \tau_{\mathrm{d} 2}\right),
$$

with $\tau_{\mathrm{d} 1}$ denoting the principal relaxation time and $\beta$ measuring the width of the (asymmetric) relaxation time distribution.

\subsection{Ultrasonic Spectra}

Figure 2 shows the ultrasonic attenuation spectrum of a long-chain alcohol in both common formats. In the frequency-normalized version, the attenuation data $\alpha / v^{2}$ indicate a two-step decrease, in correspondence with a shoulder and a relative 
maximum in the excess-attenuation-per-wavelength data, thus featuring two relaxation regions. Excess-attenuation-per-wavelength is defined as

$$
(\alpha \lambda)_{\mathrm{exc}}=\left(\alpha-\alpha_{\mathrm{asy}}\right) \lambda
$$

where $\alpha$ is the sonic attenuation coefficient, $\lambda=c_{\mathrm{s}} / \nu$ is the wavelength of the sonic field, $c_{\mathrm{s}}$ is the sound velocity of the sample, and $\alpha_{\text {asy }}=\alpha(v \rightarrow \infty)$. For non-metallic liquids the latter is given by $[15,16]$

$$
\alpha_{\text {asy }}=\frac{2 \pi^{2} v^{2}}{\rho c_{\mathrm{s}}^{3}}\left(\frac{4}{3} \eta_{\mathrm{s}}(v)+\eta_{v}(v)\right) .
$$

In this equation $\rho$ denotes the density, $\eta_{\mathrm{s}}$ is the shear viscosity, and $\eta_{v}$ is the volume viscosity of the sample. The latter is related to the curl-free part of the sonic field. At high frequencies the $\alpha / v^{2}$ data of $n$-dodecanol (Fig. 2) become smaller than the contribution,

$$
\left(\frac{\alpha}{v^{2}}\right)_{\eta_{\mathrm{s}} 0}=\frac{8 \pi^{2}}{3 \rho c_{\mathrm{s}}^{3}} \eta_{\mathrm{s}}(\nu=0)
$$

from the static shear viscosity, thus revealing not just a volume viscosity but also a shear viscosity relaxation within the frequency range under consideration. The relaxation behavior of the ultrasonic attenuation coefficient can be well represented by a sum of two Debye terms:

$$
\alpha / v^{2}=A_{\alpha 1} /\left(1+\left(\omega \tau_{\alpha 1}\right)^{2}\right)+A_{\alpha 2} /\left(1+\left(\omega \tau_{\alpha 2}\right)^{2}\right)+\alpha_{\text {asy }} / \nu^{2}
$$

with relaxation times $\tau_{\alpha 1}$ and $\tau_{\alpha 2}$ and relaxation amplitudes $A_{\alpha 1}$ and $A_{\alpha 2}$, respectively.

\subsection{Shear Viscosity Spectra}

The frequency-dependent complex shear viscosity [14]

$$
\eta_{\mathrm{s}}(v)=\eta_{\mathrm{s}}^{\prime}(v)-\mathrm{i} \eta_{\mathrm{s}}^{\prime \prime}(v)=Z_{\mathrm{s}}(v) /(2 \pi \rho v)
$$

of $n$-dodecanol, as obtained from measurements of the complex shear impedance $Z_{s}(v)$, is shown in Fig. 3 as a function of frequency and also in a complex plane representation. As suggested by the ultrasonic attenuation data, in the $\mathrm{MHz}$ frequency range the shear viscosity is in fact subject to relaxation behavior. Within the limited measurement frequency range between some $\mathrm{MHz}$ and about $100 \mathrm{MHz}$, the shear viscosity of the alcohol can be analytically well described by a Debye relaxation term,

$$
\eta_{\mathrm{s}}(v)=\eta_{\mathrm{s}}(\infty)+\left(\eta_{\mathrm{s}}(0)-\eta_{\mathrm{s}}(\infty)\right) /\left(1+\mathrm{i} \omega \tau_{\mathrm{s}}\right)
$$

with a discrete relaxation time $\tau_{\mathrm{s}}$ and with extrapolated high and low frequency values $\eta_{\mathrm{s}}(0)$ and $\eta_{\mathrm{s}}(\infty)$, respectively. 
Fig. 3 Real part $\left(\eta_{s}^{\prime}(v), \bullet\right)$ and negative imaginary part $\left(\eta_{s}^{\prime \prime}(v), \mathbf{\Lambda}\right)$ of the complex shear viscosity of $n$-dodecanol at $25^{\circ} \mathrm{C}$ displayed as a function of frequency. A complex plane representation of data (o) is given in the inset. The lines are graphs of the Debye-type relaxation function defined by Eq. 8 with parameter values found by a fitting procedure

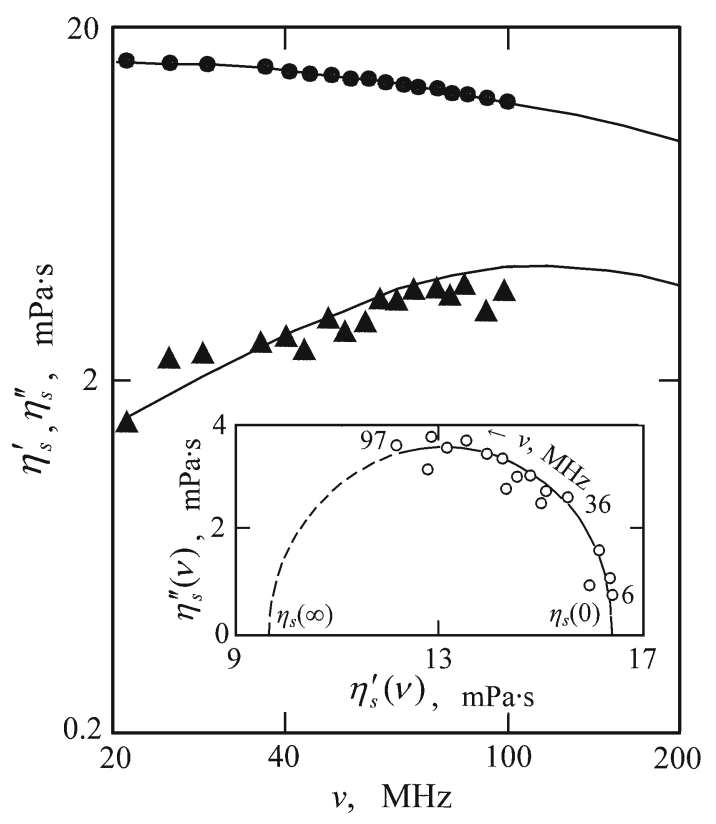

\section{Discussion}

\subsection{Terminal Hydroxy Group Rotation, Wait-and-Switch Model}

The parameter values of the high-frequency terms in the dielectric relaxation functions of alcohols and their mixtures are still subject to some discussion (e.g., [17]). Nevertheless, it is widely accepted now that term "d2" in Eqs. 2 and 3, with relaxation times between about 1 ps (methanol) and 20 ps (dodecanol), is due to the reorientational motions of single-hydrogen-bonded dipolar hydroxy groups [12,18]. At room temperature most alcohols form chainlike $\mathrm{H}$-bonded clusters with about $14 \%$ of the $\mathrm{OH}$-groups being single-bonded end groups of the structures [19,20]. Evidently, the terminal $\mathrm{OH}$-groups reorientate faster than double-H-bonded groups of the chains.

Reorientational motions of both types of hydrogen-bonded groups, i.e., singlebonded end groups and double-bonded $\mathrm{OH}$-groups within the clusters, has been discussed in terms of a "wait-and-switch" model of associating liquids [12], as originally proposed for alcohols by Sagal [21]. Within the framework of this model, the dominating part of the dielectric relaxation time is the period for which a dipole has to wait until a partner for a new hydrogen bond appears in a suitable position. Presence of this partner lowers the activation energy barrier and thus facilitates reorientation [22]. The reorientational motion itself takes a time as small as about $0.1 \mathrm{ps}[19,20,23]$ and thus resembles a switching of the dipole direction. As the concentration of single- $\mathrm{H}-$ bonded $\mathrm{OH}$-groups is significantly smaller than that of the double-bonded hydroxy groups, term "d2" with amplitude $A_{\mathrm{d} 2}$ smaller than $A_{\mathrm{d} 1}$ [12] is assumed to be due to the 
Fig. 4 Relative concentration $c_{\mathrm{d} 2} / c_{\mathrm{OH}}$ of single H-bonded hydroxy groups versus mole fraction $x_{\mathrm{OH}}$ of alcohol for mixtures of normal alcohols $\left(\mathrm{CH}_{3}\left(\mathrm{CH}_{2}\right)_{(n-1)} \mathrm{OH}\right)$ with $n$-alkanes

$\left(\mathrm{CH}_{3}\left(\mathrm{CH}_{2}\right)_{(m-2)} \mathrm{CH}_{3}\right)$ at $25^{\circ} \mathrm{C}$

$(n, m=3,7, \mathbf{\Delta} ;=5,7$,

$\mathbf{\nabla} ;=6,7, \mathbf{\square} ;=7,5$,

$\bullet$; = 12, 7, ; [12]). The inset

shows ultrasonic relaxation times $\tau_{\alpha 2}$ of the high-frequency term of alcohol spectra [14] and relaxation times $\tau_{\alpha}$ of the

Debye-type relaxation of alkane spectra at $25^{\circ} \mathrm{C}$ as a function of number of methyl groups [24]. Lines are drawn to guide the eye

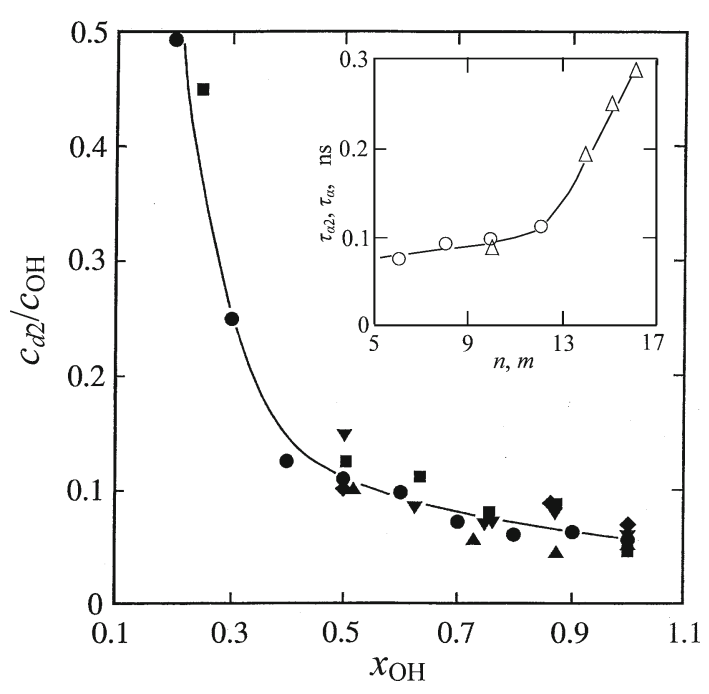

terminal $\mathrm{OH}$-groups and term "d1" is assigned to the multiply $\mathrm{H}$-bonded $\mathrm{OH}$-groups within clusters.

For some alcohol/alkane mixtures, the ratio $c_{\mathrm{d} 2} / c_{\mathrm{OH}}$ of the single-hydrogen-bonded $\mathrm{OH}$-group concentration and the total $\mathrm{OH}$-group concentration is shown for some monohydric alcohol $/ n$-alkane mixtures in Fig. 4 . These data have been calculated from the relaxation amplitudes $A_{\mathrm{d} 2}$ and $A_{\mathrm{d} 1}$, assuming identical orientation correlation factors for single- and multiply-H-bonded alcohol molecules [12]. Evidently, a rather uniform behavior follows from the data for different mixtures: the relative concentration of single-H-bonded $\mathrm{OH}$ groups increases when the alcohol structures are increasingly disturbed by the addition of alkane. This increase in the single-H-bonded alcohol content is accompanied by a decrease in the orientation correlation of dipole directions as follows from the static permittivity data of the mixtures [12].

\subsection{Alkyl Chain Isomerization}

Also shown in Fig. 4 are the high-frequency relaxation times $\tau_{\alpha 2}$ of the ultrasonic attenuation spectra of some alcohols. The $\tau_{\alpha 2}$ data nicely fit to the relaxation time values for $n$-alkanes given in the diagram as well. The analogy in the chain-length dependence of relaxation times has suggested the high-frequency ultrasonic relaxation term to be correlated with the chain conformational isomerization of the alcohol molecules. This assignment is confirmed by ultrasonic measurements of mixtures of $n$-dodecanol with $n$-hexadecane which reveal a unimolecular (zero-order) reaction $\mathrm{A} \leftrightarrow \mathrm{A}^{*}$ between alcohol conformations $\mathrm{A}$ and $\mathrm{A}^{*}$, respectively. The strong dependence of the relaxation times upon the chain length suggests a collective phenomenon, as considered by the coupled torsional oscillator model $[25,26]$ of chain isomerization. This model predicts a series of relaxation terms, each one related to a mode of chain motions. Evaluating the " $\alpha 2$ " term of the ultrasonic spectra, the first normal mode, the 
one with the smallest relaxation frequency, has been assumed. It reflects the transition from the all-trans conformation of a chain to a conformation with a mean number or rotated $\mathrm{C}-\mathrm{C}$ bonds. Identifying the relaxation rate,

$$
\nu_{1}=2 v_{\mathrm{t} 0} \sin ^{2}\left(\pi / 2 n_{\mathrm{t} 0}\right) \exp \left(-\Delta G^{\#} / R T\right)
$$

of this first normal mode with $\tau_{\alpha 2}^{-1}$ yields the Gibbs free energy of activation $\Delta G^{\#}$ for the mode of torsional oscillations. In Eq. 10, $v_{\mathrm{t} 0}$ is the characteristic frequency for the rotation of an individual $\mathrm{C}-\mathrm{C}$ bond, $n_{\mathrm{t} 0}$ is the number of torsional oscillator par alkyl chain, and $R$ is the universal gas constant. Using $v_{\mathrm{t} 0}=8.5 \mathrm{THz}$ as reported for ethane [27] and $n_{\mathrm{t} 0}=n / 3$, where $n$ is the number of methyl groups per alkyl chain, reasonable values between $15.9 \mathrm{~kJ} \cdot \mathrm{mol}^{-1}\left(n\right.$-hexanol) and $13.8 \mathrm{~kJ} \cdot \mathrm{mol}^{-1}$ ( $n$-dodecanol) follow [14], in fair agreement with Gibbs free energies of activation for $n$-alkanes (e.g., $\Delta G^{\#}=14.1 \mathrm{~kJ} \cdot \mathrm{mol}^{-1}, n$-dodecane [24]). The chain isomerization is associated with a high-frequency shear viscosity relaxation [24]. Estimation of the corresponding relaxation amplitude $\Delta \eta_{\mathrm{s} 2}$ from the ultrasonic parameters, according to

$$
\Delta \eta_{\mathrm{s} 2}=3 A_{\alpha 2} \tau_{\alpha 2} \rho c_{\mathrm{s}}^{2} /(4 \pi)
$$

yields shear viscosities $\eta_{\mathrm{s}}(\infty)-\Delta \eta_{\mathrm{s} 2}$ on the order of $1 \mathrm{mPa} \cdot \mathrm{s}$ [24] at frequencies well above the relaxation region $(v>5 \mathrm{GHz})$.

\subsection{Dominating Relaxation, Multiply-H-Bonded Cluster Molecule Motions}

For the principal relaxation terms, relaxation times $\tau_{\eta 1}\left(=\tau_{\alpha 1}\right)$ of the longitudinal viscosity and of the longitudinal dielectric relaxation times $\tau_{\mathrm{dl}}=\tau_{\mathrm{d} 1} \varepsilon(\infty) / \varepsilon(0)$, assigned to the motions of multiply H-bonded molecules, are displayed versus concentration $c_{\mathrm{OH}}$ of hydroxy groups in the main part of Fig. 5. The data for alcohols and for alcohol/alcohol mixtures (full symbols) strongly support the idea of the wait-andswitch model. With increasing content of hydroxy groups, the probability density for the availability of a suitable partner for the formation of a new hydrogen bond increases. Therefore, the waiting periods and, consequently, the relaxation times decrease. This view is confirmed by the dielectric relaxation times $\tau_{\mathrm{d} 1}$ of alcohol/water mixtures which also significantly decrease with concentration $c_{\mathrm{HB}}$ of hydrogen-bonding groups or molecules (Fig. 5, inset).

As the approach of a new hydrogen-bond partner is governed by diffusion, the alkyl chain length plays a significant role in the waiting period. The molar enthalpy of activation $\Delta H_{1}^{\#}$ of the dominating dielectric relaxation of normal alcohols $\mathrm{CH}_{3}\left(\mathrm{CH}_{2}\right)_{(n-1)} \mathrm{OH}$ can be represented by a sum of three terms [28],

$$
\Delta H_{1}^{\#}=\Delta H_{\mathrm{OH}}^{\#}+(n-1) \Delta H_{\mathrm{CH}_{2}}^{\#}+\Delta H_{\mathrm{CH}_{3}}^{\#}
$$

with $\Delta H_{\mathrm{CH}_{2}}^{\#}=3.5 \mathrm{~kJ} \cdot \mathrm{mol}^{-1}$ at $n \leq 8$. At higher $n$, folding of the alkyl chains seems to lead to a small reduction in the intermolecular interactions and thus to smaller 
Fig. 5 Relaxation times

$\tau_{\eta 1}\left(=\tau_{\alpha 1}\right)$ and

$\tau_{\mathrm{dl} l}\left(=\tau_{\mathrm{d} 1} \varepsilon(\infty) / \varepsilon(0)\right)$ versus

the concentration $c_{\mathrm{OH}}$ of hydroxy groups for alcohols

$\left(\tau_{\mathrm{dl}}, \bullet[12] ; \tau_{\eta \mathrm{l}}, \boldsymbol{\Delta}[14]\right)$ and

ethanol $/ n$-hexanol mixtures

$\left(\tau_{\mathrm{dl}}, \mathbf{[ 2 8 ]}\right)$, as well as for mixtures of normal alcohols with alkanes $\left(\circ, \tau_{\mathrm{dl}}, n\right.$-heptane [12]; $\Delta, \tau_{\eta 1}, n$-heptane [29], $\square, \tau_{\eta 1}, n$-tetradecane [29]) at $25^{\circ} \mathrm{C}$. For $n$-alcohols $(\bullet)$, water $(\diamond)$, and alcohol/water mixtures $(\diamond)$, the relaxation time of the dominating dielectric relaxation process is displayed versus the concentration $c_{\mathrm{HB}}$ of hydrogen-bonding groups and molecules in the inset [30]

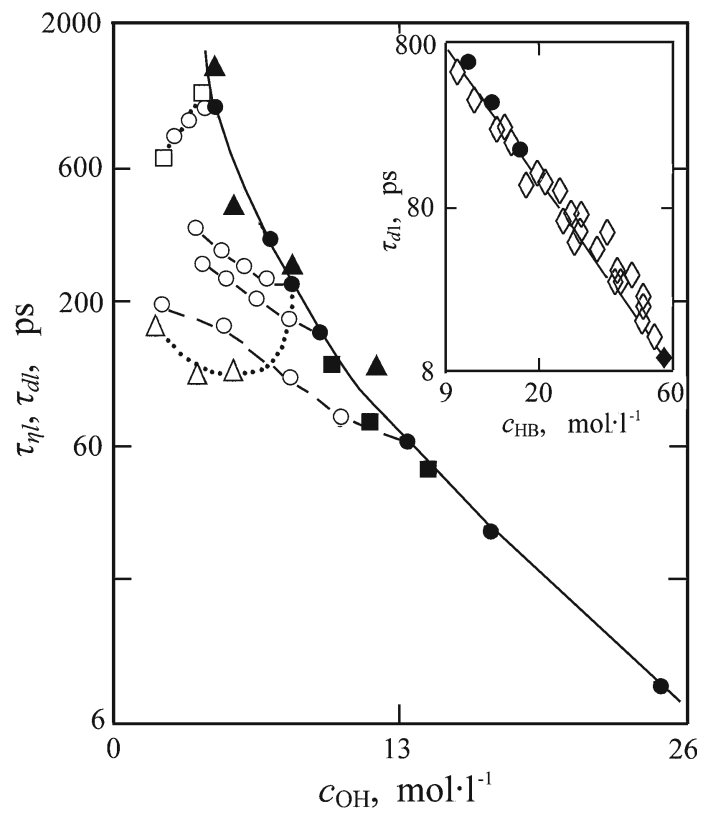

$\Delta H_{\mathrm{CH}_{2}}^{\#}$ values [28]. At a first glance, the rise in the $\Delta H_{1}^{\#}$ values at increasing alkyl chain length might be associated with increasing Coulomb interactions and thus stronger hydrogen bonds when the static permittivity of the alcohols decreases. However, infrared spectroscopy did not indicate changes in the hydrogen-bond energy within the series of normal alcohols [31].

This analysis is consistent with the previous conclusion that the major relaxation is due to a dynamic equilibrium between (predominantly) clustered states [32]. The relaxation spectra, however, do not allow for statements on possible specific structures, such as dimers, as had been suggested from infrared measurements [33].

The correlation of the longitudinal dielectric relaxation times with the ultrasonic relaxation times is a noticeable result. The former is the characteristic time of dipole reorientation at a constant charge distribution for a given site. This reorientation appears thus to be associated with a volume change. Obviously, the significantly larger experimental relaxation times $\tau_{\mathrm{d} 1}=\tau_{\mathrm{dl}} \varepsilon(0) / \varepsilon(\infty)$ include the collective redistribution of the electrical charges.

\subsection{Clustering, Alcohol-Alkane Mixtures}

Relaxation times $\tau_{\mathrm{dl}}$ and $\tau_{\eta 1}$ of alcohol/alkane mixtures display different behaviors as a function of the hydroxy group concentration (Fig. 5). They either increase less strongly with decreasing $c_{\mathrm{OH}}$ than the data for pure alcohols or alcohol-alcohol mixtures, are almost concentration independent, or even decrease. Likely, two types of mixtures of alkanes, $\mathrm{CH}_{3}\left(\mathrm{CH}_{2}\right)_{(m-2)} \mathrm{CH}_{3}$, with normal alcohols, $\mathrm{CH}_{3}\left(\mathrm{CH}_{2}\right)_{(n-1)} \mathrm{OH}$, exist. At $m<n$ the non-polar alkane molecules seem to be predominantly solved in 
hydrocarbon regions of H-bonded alcohol structures. In such a situation, because of their elevated flexibility, alkanes tend to increase the mobility of alcohol molecules. Within the framework of the wait-and-switch model, the enhanced diffusion coefficient of the alcohol molecules leads to a shorter waiting period and thus to a smaller relaxation time.

For systems with alkanes longer than the alkyl group of the normal alcohol $(m>n)$, incorporation of the non-polar molecules in the hydrocarbon part of the alcohol structures is unlikely. Rather the mixtures will be inclined to form a micro-heterogeneous structure with sub-phases of different compositions. At high alkane content, alcohol structures, with some similarity to inverse micelles, are being supposedly formed. Such structures involve regions of a high $\mathrm{OH}$-group concentration in which the probability density for the occurrence of a new suitable hydrogen-bond partner, and thus the waiting period in the relaxation time, is again small.

\subsection{Concentration Fluctuations, Mixtures of Alcohols and Water}

Mixtures of alcohols with water often reveal ultrasonic attenuation spectra which are broader than a Debye-type relaxation term with a discrete relaxation time. An example is shown in the inset of Fig. 6. A series of spectra for mixtures of short-chain alcohols has been discussed including relaxation functions that model fluctuations in the local concentration [34] and visco-inertial as well as thermal conductivity effects due to a micro-heterogeneous liquid structure [35]. A favorable description of the alcohol/water spectra is obtained in terms of a more recent unifying model of noncritical concentration fluctuations [36]. This model is based on the assumption that

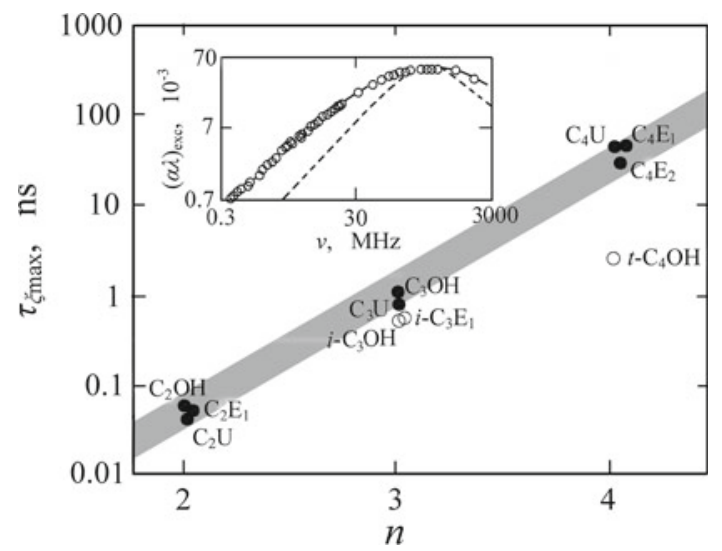

Fig. 6 Maximum characteristic relaxation times $\tau_{\xi \max }$ versus number $n$ of carbon atoms per alkyl group for some mixtures of water with alcohols, poly(ethylene glycol) monoalkyl ethers, and derivatives of urea [36]: $\mathrm{C}_{2} \mathrm{OH}$, ethanol; $\mathrm{C}_{2} \mathrm{E}_{1}$, ethoxyethanol; $\mathrm{C}_{2} \mathrm{U}$, ethylurea; $\mathrm{C}_{3} \mathrm{OH}, n$-propanol; $i$ - $\mathrm{C}_{3} \mathrm{OH}$, iso-propanol; $i-\mathrm{C}_{3} E_{1}$; iso-propoxyethanol; $\mathrm{C}_{3} \mathrm{U}, n$-propylurea; $t-\mathrm{C}_{4} \mathrm{OH}$, tert-butanol; $\mathrm{C}_{4} \mathrm{E}_{1}$, 2-butoxyethanol; $\mathrm{C}_{4} \mathrm{E}_{2}, 2$-(2-butoxyethoxy)ethanol; $\mathrm{C}_{4} \mathrm{U}, n$-butylurea. In the inset the excess-attenuation-per-wavelength spectrum of a solution of $n$-propanol $\left(4.9 \mathrm{~mol} \cdot \mathrm{L}^{-1}, 25^{\circ} \mathrm{C}\right.$ [34]) in water is displayed. Dashed line is the graph of a Debye relaxation term 
thermal equilibrium is reached via two parallel pathways. One is provided by a rate process with a discrete relaxation time $\tau_{0}$, the other one by diffusion. The latter is controlled by the relaxation rate $\tau_{\xi}^{-1}=2 D / \xi^{2}$, where $D$ denotes the mutual diffusion coefficient and $\xi$ is the fluctuation correlation length. With $q=|\vec{q}|$ denoting the modulus of the wave vector, the excess attenuation per wavelength is given by the integral equation,

$$
(\alpha \lambda)_{\mathrm{exc}}=Q \int_{0}^{\infty} \frac{q^{2} \mathrm{~d} q}{\left(1+0.164 q \xi+0.25(q \xi)^{2}\right)^{2}} \frac{\omega \tau_{g}}{1+\left(\omega \tau_{g}\right)^{2}} .
$$

Here $Q$ is an amplitude and $\tau_{g}=\left(D q^{2}+\tau_{0}^{-1}\right)^{-1}$.

As result of application of the model relaxation spectral function (Eq. 13), $\tau_{\xi \max }$ data as displayed in Fig. 6 have been found. For each aqueous system these data represent the maximum $\tau_{\xi}$ value within a concentration series. For mixtures of water with monohydric alcohols, poly(ethylene glycol) monoalkyl ethers, and derivatives of urea, the $\tau_{\xi \max }$ values reveal some interesting trends. The maximum relaxation times strongly increase with the number of carbon atoms per alkyl group, indicating the vigorous promotion of concentration fluctuations by hydrophobic groups of the non-aqueous constituent. The uniform behavior for solutes with an unbranched alkyl group evidences an unexpectedly minor role of the hydrophilic group of the organic constituent. Quite remarkably, however, effects of alkyl group isomerization are of paramount importance for the formation of a micro-heterogeneous structure of mixtures of organic constituents with water. This finding is illustrated by the small $\tau \xi \max$ value for mixtures of tert-butanol with water (Fig. 6). Another example involves solutions of tetramethylurea with $\tau_{\xi \max }(=10 \mathrm{ps}$ [36]) on the order of molecular reorientation times. This value contrasts $\tau_{\xi \max }=40 \mathrm{~ns}$ for isomeric $n$-butylurea (Fig. 6) that clearly reveals long-ranging correlations in the fluctuations. The favored promotion of a microheterogeneous liquid structure by an unbranched alkyl group suggests that hydrophobic interactions lead to a clustering between these groups and thus to some kind of pre-micellar oligomeric structures in water.

Open Access This article is distributed under the terms of the Creative Commons Attribution Noncommercial License which permits any noncommercial use, distribution, and reproduction in any medium, provided the original author(s) and source are credited.

\section{References}

1. L.-M. Wang, S. Shahriari, R. Riechert, J. Phys. Chem. B 109, 23255 (2005)

2. R. Behrends, K. Fuchs, U. Kaatze, Y. Hayashi, Y. Feldman, J. Chem. Phys. 124, 144512 (2006)

3. T. El Goresy, R. Böhmer, J. Chem. Phys. 128, 154520 (2008)

4. A.D. Vyas, V.A. Rana, S.P. Bhatnagar, V.M. Vashisth, Indian J. Pure Appl. Phys. 46, 521 (2008)

5. K. Bebek, A. Strugała, Eur. Phys. J. Spec. Topics 154, 257 (2008)

6. A.C. Kumbharkhane, M.N. Shinde, S.C. Mehrotra, N. Oshiki, N. Shinyashiki, S. Yagihara, S. Sudo, J. Phys. Chem. A 113, 10196 (2009)

7. E. Zorębski, Int. J. Thermophys. 31, 143 (2010)

8. U. Kaatze, R. Behrends, K. Roden, J. Chem. Phys. 133, 094508 (2010) 
9. U. Kaatze, M. Schäfer, R. Pottel, Z. Phys. Chem. (Munich) 165, 103 (1989)

10. J. Barthel, K. Bachhuber, R. Buchner, H. Hetzenauer, Chem. Phys. Lett. 165, 369 (1990)

11. R. Buchner, J. Barthel, J. Mol. Liq. 52, 131 (1992)

12. S. Schwerdtfeger, F. Köhler, R. Pottel, U. Kaatze, J. Chem. Phys. 115, 4186 (2001)

13. R.H. Cole, D.W. Davidson, J. Chem. Phys. 20, 1389 (1952)

14. R. Behrends, U. Kaatze, J. Phys. Chem. A 105, 5829 (2001)

15. K.F. Herzfeld, T.A. Litovitz, Absorption and Dispersion of Ultrasonic Waves (Academic Press, Inc, New York, 1959)

16. J.K. Bhattacharjee, U. Kaatze, S.Z. Mirzaev, Rep. Prog. Phys. 73, 066601 (2010)

17. J.T. Kindt, C.A. Schmuttenmaer, J. Phys. Chem. 100, 10373 (1996)

18. T. Sato, A. Chiba, R. Nozaki, J. Mol. Liq. 101, 99 (2002)

19. W.L. Jørgensen, J. Chem. Phys. 90, 1276 (1986)

20. L. Saiz, J.A. Padró, E. Guàrdia, J. Phys. Chem. B 101, 78 (1997)

21. M.W. Sagal, J. Chem. Phys. 36, 2437 (1962)

22. F. Sciortino, A. Geiger, H.E. Stanley, J. Chem. Phys. 96, 3857 (1992)

23. I. Ohmine, H. Tanaka, Chem. Rev. (Washington, DC) 93, 2545 (1993)

24. R. Behrends, U. Kaatze, J. Phys. Chem. A 104, 3269 (2000)

25. A.V. Tobolsky, J. Polym. Sci., Part A: Polym. Chem. 6, 1177 (1968)

26. A.V. Tobolsky, D.B. Du Pré, Adv. Polym. Sci. 6, 103 (1969)

27. K.S. Pitzer, J. Chem. Phys. 12, 310 (1944)

28. P. Petong, R. Pottel, U. Kaatze, J. Phys. Chem. A 103, 6114 (1999)

29. U. Kaatze, R. Behrends, Chem. Phys. Lett. 510, 67 (2011)

30. U. Kaatze, R. Behrends, R. Pottel, J. Non-Cryst. Solids 305, 19 (2002)

31. L. Wilson, R. Bioca De Atencastro, C. Sandorfy, Can. J. Chem. 63, 40 (1985)

32. C. Dugue, J. Emery, R.A. Pethrick, Mol. Phys. 42, 1453 (1981)

33. M. Tabellout, P. Lanceleur, J.R. Emery, D. Hayward, R.A. Pethrick, J. Chem. Soc. Faraday Trans. 86, 1493 (1990)

34. M. Brai, U. Kaatze, J. Phys. Chem. 96, 8946 (1992)

35. U. Kaatze, M. Brai, K. Menzel, Ber. Bunsen-Ges. Phys. Chem. 98, 1 (1994)

36. A. Rupprecht, U. Kaatze, J. Phys. Chem. 103, 6485 (1999) 